\title{
Effectiveness of epidural versus alternate analgesia for pain relief after radical prostatectomy and correlation with biochemical recurrence in men with prostate cancer
}

\author{
This article was published in the following Dove Press journal: \\ Research and Reports in Urology \\ 18 October 2013 \\ Number of times this article has been viewed
}

\author{
Maria C Mir' \\ Binoy Joseph' \\ Rona Zhao' \\ Damien M Bolton' \\ Dennis Gyomber' \\ Nathan Lawrentschuk ${ }^{1,2}$ \\ 'University of Melbourne, Department \\ of Surgery, Austin Hospital, \\ Melbourne, VIC, Australia; ${ }^{2}$ Ludwig \\ Institute for Cancer Research, Austin \\ Hospital, Melbourne, VIC, Australia
}

\begin{abstract}
Objectives: Our objectives were to analyze the effectiveness of epidural anesthesia in patients who underwent open retropubic radical prostatectomy (RRP) at our institution over the past decade, and to examine subsequent oncologic outcomes, comparing those receiving with those not receiving epidural anesthesia.

Methods: A comprehensive database of all patients undergoing RRP from November 1996 to December 2006 was analyzed; 354 patients underwent RRP at our institution and were divided into those receiving or not receiving an epidural. An independent pain management team scoring technical success found epidural technique to be consistent. Oncological outcome was an endpoint of our study, comparing both analysis groups. We classed prostate-specific antigen (PSA) recurrence after RRP as a serum PSA $\geq 0.2 \mathrm{ng} / \mathrm{mL}$ at any stage of postoperative follow-up. Complications were recorded to 30 days using the modified Clavien system, and full statistical analyses were undertaken.
\end{abstract}

Results: Records were available for 239 men; we observed a decreased trend in the use of epidural for pain management, along with a decrease in average hospital stay and an overall epidural success rate of $64 \%$. When dividing data into RRP with and without epidural, we found a median hospital stay of 7 days for patients receiving an epidural compared with 6 days for those not receiving an epidural. The differences were statistically significant $(P<0.048)$ and remained so after adjusting for complications $(P<0.0001)$. Regarding oncological outcome, PSA recurrence was further analyzed in this cohort. Percentage of recurrence was higher $(14.8 \%)$ for patients receiving an epidural than for the non-epidural group (4.8\%). The differences were statistically significant $(P=0.012)$.

Conclusion: Epidural analgesia increased length of hospital stay and technical problems related to the epidural. Furthermore, men receiving an epidural showed an increased recurrence of PSA. In light of our findings, epidurals are probably not indicated for men undergoing RRP. However, as minimally invasive techniques are becoming more widespread, and epidural analgesia is being used less frequently, large randomized controlled trials to definitively support our hypotheses are unlikely to be undertaken.

Keywords: prostate neoplasm, surgery, prostatectomy, analgesia, epidural

\section{Introduction}

Prostate cancer $(\mathrm{PCa})$ remains the second leading cause of cancer death in men. One man in six will get PCa during his lifetime, whilst one in 36 will die of this disease. However, PCa mortality has been declining steadily over the last 2 decades, which may be attributed to multiple reasons, including advances in treatment and early detection
Correspondence: Nathan Lawrentschuk University of Melbourne, Department of Surgery and Ludwig Institute for Cancer Research, Austin Hospital, Studley Road, 3084 Heidelberg, VIC, Australia Email urologiconcologist@gmail.com submit your manuscript | www.dovepress.com

Dovepress

http://dx.doi.org/10.2147/RRU.S49219 
of the disease. ${ }^{1,2}$ The Australian health care system (a mixture of public and private systems) certainly embraced surgery for $\mathrm{PCa}$ and recently has witnessed a rapid evolution in the surgical treatment of localized $\mathrm{PCa}$ and its perioperative management. Despite recent advances in surgical techniques gaining traction, including laparoscopic and robotic approaches, open retropubic radical prostatectomy (RRP) is still being performed in relatively large numbers worldwide, remaining the 'gold standard' for this surgery ${ }^{3}$ (particularly in the public teaching system, where robots are essentially unavailable), and this is likely to remain the case for at least the next decade.

The Australian health system has been under pressure to reduce hospital stays following surgery. Regarding RRP, postoperative pain management has dominated length of stay and so remains one of the most important aspects of perioperative management. Pain has been demonstrated to impact on the performance of the human body and its systems, including recovery time, recurrence of disease, and overall patient well-being. ${ }^{4}$ Intravenous patient-controlled analgesia with or without local anesthetic wound infiltration, including intra-wound catheters, non-steroidal anti-inflammatory agents, opioids, regular paracetamol, and epidural analgesia, are the most commonly used techniques for management of postoperative pain after RRP in Australian institutions. ${ }^{5}$

The uptake of epidural anesthesia and analgesia was swift in Australia in the 1990s, particularly for lower abdominal surgery and obstetrics, and was highlighted by the completion of the large Multicentre Australian Study of Epidural Anaesthesia (MASTER) trial at the time. ${ }^{5}$ Epidural management involves continuous administration of a local anesthetic-opioid combination into the epidural space via an indwelling catheter. However, epidural anesthesia is relatively expensive, and requires special equipment, training, and close nurse observation to ensure proper management. Furthermore, epidural anesthesia has contraindications and complications that require follow-up, such as bleeding disorders, local sepsis, local anesthetic allergy, or blood pressure lability. Data on effectiveness of this pain management approach for RRP are minimal.

Another aspect, apart from efficacy of epidural anesthesia and analgesia, is that of impact on oncological outcome. Available animal data suggest that regional anesthesia helps to preserve effective defenses against tumor progression by attenuating the surgical stress response, reducing general anesthesia requirements, and sparing postoperative opioids. ${ }^{6}$ However, again, sparse human data on this topic exist, particularly for RRP. Exadaktylos et $\mathrm{al}^{7}$ suggested that paravertebral anesthesia for breast cancer surgery was associated with a 4-fold reduced risk of recurrence or metastasis during a 4-year follow-up.

Against this background, and with open surgery still being performed for PCa, we analyze the effectiveness of epidural anesthesia in relation to patients who underwent RRP at our institution over the past decade. Furthermore, we examine subsequent oncologic outcomes, comparing those receiving epidural anesthesia and those not receiving epidural anesthesia.

\section{Material and methods \\ Patient population}

A comprehensive prospective database of all patients undergoing RRP from November 1996 to December 2006 was constructed according to institutional ethical committee standards. A total of 354 patients underwent RRP at our institution during this time. The data were then retrospectively analyzed, with electronic medical records consulted if required. The following data were obtained: demographic characteristics, tumor size, Gleason score, prostate-specific antigen (PSA) level, pelvic node disease, post-surgery radiation or hormone therapy. Seven patients were excluded from the study due to incomplete data. Oncologic outcome comparing both analysis groups was an endpoint in our study. We stated PSA recurrence after RRP as serum PSA $\geq 0.2 \mathrm{ng} / \mathrm{mL}$ at any stage of postoperative follow-up (minimum 3.5 years). Patients underwent a bilateral pelvic standard lymphadenectomy if their D'Amico disease risk classification was intermediate or high. ${ }^{8}$

Patients were first divided into one of two groups, according to postoperative analgesia used: epidural versus nonepidural (ie, use of patient-controlled analgesia and wound infiltration with local anesthetic). Complications affecting the length of hospital stay were identified as a potential confounding factor, and if recorded, these records were selectively removed for sub-analysis. Patients who underwent laparoscopic surgery were also identified and excluded $(n=70)$. Thus, analysis was carried out not only for the total population, but also for the open groups without any complications, in an attempt to compare homogeneous data.

\section{Epidural technique}

The protocol for epidural technique was followed according to our institution standard procedures. Epidural complications/ failures were also recorded. For the purpose of this study, we considered epidural treatment successful if the infusion was used until there was no further need for epidural. Alter- 
natively, it was considered a failure if the infusion was discontinued prematurely due to problems with the catheter or treatment that could not be controlled through intervention (eg, increasing dose, altering analgesic agents in epidural, etc) as recorded by the independent pain management team. Similar selection criteria for epidural failure was used by Ballantyne. ${ }^{9}$ The year 2004 was chosen for comparative analysis as the 'cutoff' in this study because this was when epidural use was at its maximum in the hospital.

\section{Complications}

Complications were recorded out to 30 days using the modified Clavien system. ${ }^{10}$ Any complications in the epidural group are reported separately (see Table 1) for clarity and comparison.

\section{Statistical analyses}

Where appropriate, demographic and clinical data were calculated with means and standard deviations. The Mann-Whitney test was used for all statistical analysis. Data were considered statistically significant at a $P$-value $<0.05$ (Microsoft $^{\circledR}$ Excel, Microsoft Corporation, and GraphPad Prism 4.0, GraphPad Software Inc, CA, USA).

\section{Results}

Of the 284 men undergoing RRP, 239 open RRP cases were available (remainder laparoscopic $n=45$ ). Our two groups (epidural versus [vs] non-epidural) demonstrated similar perioperative levels of PSA, Gleason score, and positive margins. Patient characteristics are described in Table 2.

We observed a decreased trend in the use of epidurals for pain management between 1996 and 2006 at our institution, along with a decrease in average length of hospital stay for patients undergoing RRP. When dividing data into epidural and non-epidural RRP, we found a median of 7 days of hospital stay for patients receiving an epidural compared with 6 days for non-epidural patients. The differences were statistically significant $(P=0.05)$.

The difference in length of hospital stay between the two groups is also statistically significant $(P<0.0001)$. After adjusting for the confounding factor of a complication, the differences still remain $(P<0.0001)$ (Figure 1). If year 2004 is considered as the cut-off point for epidural training, we observe that the trend persists (Figure 2).

When analyzing epidural success per se, we found a $64 \%$ success versus a $36 \%$ failure rate. Further analysis of the independent pain management team notes indicated

Table I Pathological characteristics of patients with retropubic radical prostatectomy and epidural with prostate-specific antigen recurrence (last six patients in bold = non-epidural)

\begin{tabular}{|c|c|c|c|c|c|c|}
\hline $\begin{array}{l}\text { PSA failure } \\
(\mathrm{ng} / \mathrm{mL})\end{array}$ & $\begin{array}{l}\text { Initial PSA } \\
(\mathrm{ng} / \mathrm{mL})\end{array}$ & TNM & $\begin{array}{l}\text { Gleason } \\
\text { score }\end{array}$ & $\begin{array}{l}\text { Margin } \\
\text { involvement }\end{array}$ & $\begin{array}{l}\text { Hormonal } \\
\text { therapy }\end{array}$ & $\begin{array}{l}\text { Radiation } \\
\text { therapy }\end{array}$ \\
\hline 0.3 & 6.7 & T2 No & 7 & Positive & No & No \\
\hline 1.2 & 10.6 & T3a N0 & 7 & Negative & Yes & Yes \\
\hline 0.3 & 9.8 & T2 No & 5 & Positive & No & No \\
\hline 0.2 & 7.7 & T3a N0 & 7 & Negative & Yes & No \\
\hline 0.2 & 15 & T2 No & 7 & Negative & No & Yes \\
\hline 0.2 & 3.1 & T2 No & 9 & Positive & Yes & Yes \\
\hline 0.3 & 6 & T2 No & 2 & Negative & No & No \\
\hline 0.7 & 12.1 & T2 No & 6 & Positive & Yes & No \\
\hline 1.2 & 14 & T3a N0 & 7 & Positive & Yes & No \\
\hline 0.2 & 16.1 & T2 No & 7 & Positive & No & No \\
\hline 0.5 & 16.6 & T2 NI & 7 & Negative & Yes & No \\
\hline 0.4 & 4.8 & T2 No & 7 & Positive & Yes & Yes \\
\hline 9.9 & 23.2 & T3a N0 & 7 & Positive & Yes & No \\
\hline 0.2 & 10 & T2 No & 7 & Positive & Yes & No \\
\hline 0.4 & 10.3 & T3b N0 & 7 & Negative & Yes & No \\
\hline 0.2 & 14.9 & T2 No & 7 & Positive & No & No \\
\hline 0.2 & 13.1 & T2 No & 3 & Positive & No & No \\
\hline 3.9 & 9 & T2 No & 9 & Negative & Yes & No \\
\hline 0.2 & 15 & T2 No & 7 & Positive & No & No \\
\hline 0.2 & 17 & T3a No & 7 & Positive & Yes & Yes \\
\hline 1.7 & 10.1 & T3a N0 & 9 & Positive & Yes & No \\
\hline 0.2 & 0.7 & T2 No & 5 & Negative & No & Yes \\
\hline 0.4 & 10.7 & T2 No & 7 & Positive & Yes & No \\
\hline
\end{tabular}

Abbreviation: PSA, prostate-specific antigen. 
Table 2 Demographic, pathological, and clinical characteristics of the study group

\begin{tabular}{lll}
\hline Characteristic & Epidural & Non-epidural \\
\hline N (239) & 115 & 124 \\
Age (years, median) & 62 & 63 \\
PSA (ng/mL) & 8.7 & 9.1 \\
TNM & & \\
TIc & 25 & 24 \\
T2 & 67 & 73 \\
T3 & 23 & 27 \\
No & 61 & 62 \\
NI & 19 & 23 \\
NX & 35 & 39 \\
Positive margins (\%) & 21 & 20 \\
Gleason & & \\
6 & 36 & 40 \\
7 & 45 & 49 \\
8 & 34 & 35 \\
Duration of surgery (minutes, mean) & 119 & 135 \\
Epidurals requiring change to PCA & 42 & - \\
Epidurals used along with PCA & 5 & - \\
Complications & & \\
Total & 22 & 29 \\
Clavien I & 11 & 14 \\
Clavien II & 8 & 10 \\
Clavien III & 2 & 3 \\
Clavien IV & 1 & 2 \\
Clavien V & - & - \\
\hline
\end{tabular}

Abbreviations: PCA, patient-controlled analgesia; PSA, prostate-specific antigen. that $98 \%$ of the epidural failures were related to technical issues.

The overall percentage of complications in relation to the anesthetic technique (Table 2) was not statistically significant (19\% epidural vs $25 \%$ non-epidural; $P=0.8$ ). Minor complications related to the epidural were recorded (Table 3), with only four of these recorded as Clavien Type 1 as per protocol (Table 2).

Regarding oncologic outcome, PSA recurrence was analyzed further in this cohort (Table 1). A higher percentage of recurrence was found in the epidural group $(17 / 115=14.8 \%)$ than in the non-epidural group $(6 / 124=4.8 \%)$. The differences were found to be statistically significant $(P=0.012)$.

\section{Discussion}

Our study describes the evolution of pain management and oncologic outcome after RRP in a single tertiary center. Our results suggest a decline in, if not cessation of, the overall use of epidural anesthesia for pain management in RRP patients. Although Rodgers et $\mathrm{al}^{11}$ have previously found the epidural to provide better pain control, in our environment, disadvantages appear to outweigh advantages of its use.

Much of the decline in epidural use resulted from the results of the Australian MASTER study, published in

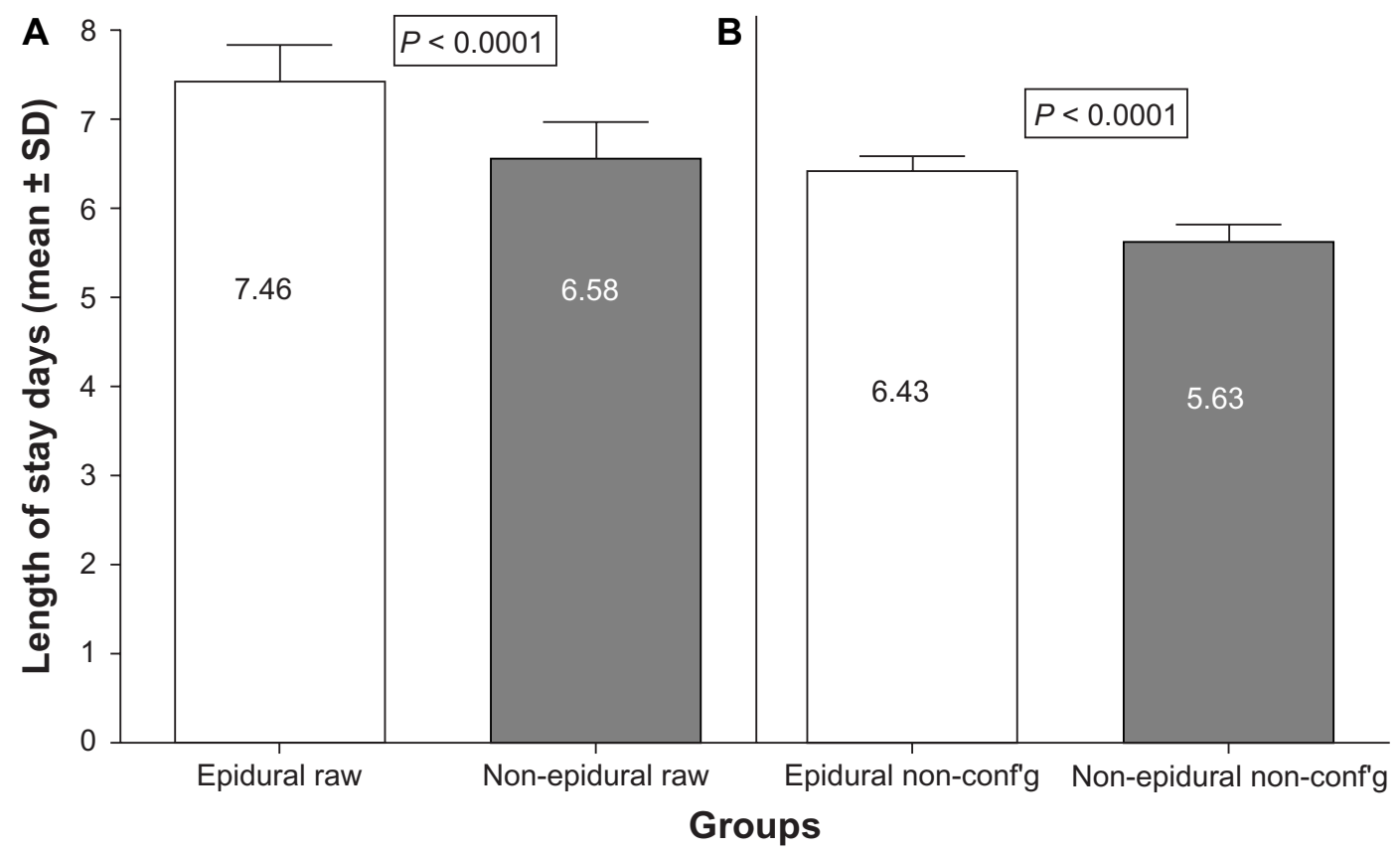

Figure I Comparison of epidural group and non-epidural group.

Notes: (A) with 'raw' data, not accounting for any confounding factors, and (B) with data taking into account confounding factors (age, TNM, PSA, Gleason score).

Abbreviations: PSA, prostate-specific antigen; SD, standard deviation; non-conf'g, non confounding. 
A

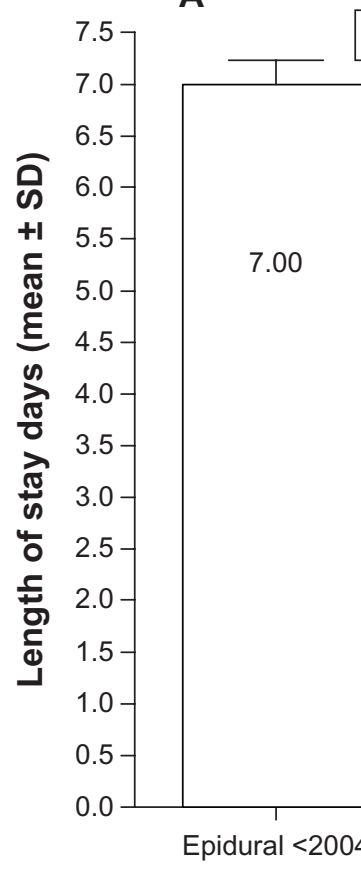

B

$P=0.05$

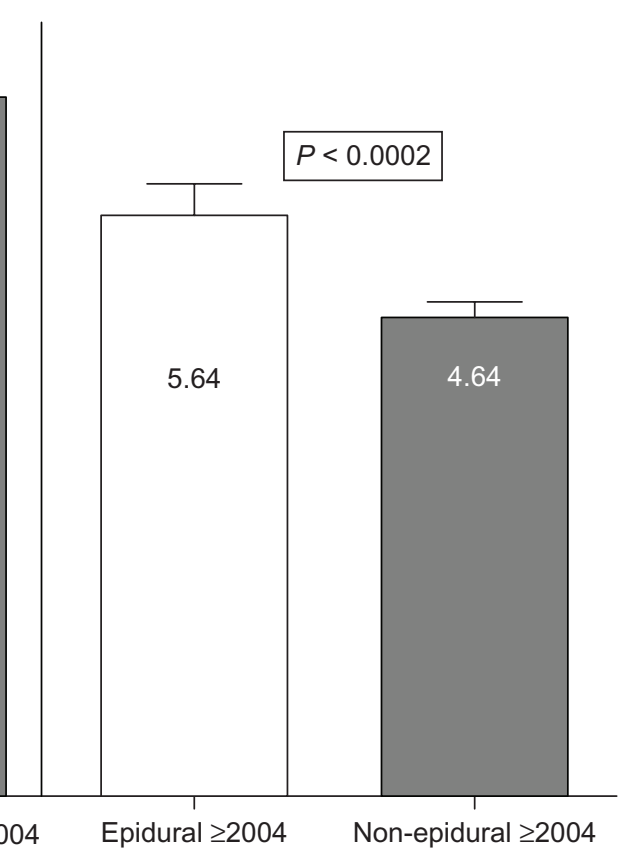

Patient group by time cohort

Figure 2 Comparison of epidural group and non-epidural group.

Notes: (A) With data prior to 2004, and (B) with data during and after 2004.

Abbreviation: SD, standard deviation.

2002. The authors analyzed 915 patients undergoing major abdominal surgery. Patients were defined as 'high-risk' with one of nine defined comorbid states, and were randomly assigned intraoperative epidural anesthesia and postoperative epidural analgesia for 72 hours, with general anesthesia (site of epidural selected to provide optimum block) or control. Mortality at 30 days was low in both groups (epidural 23 [5.1\%], control 19 [4.3\%]; $P=0.67$ ). Only

Table 3 Local complications due to the epidural only in the epidural sub-group $(n=115)$

\begin{tabular}{ll}
\hline Complication & $\mathbf{N}$ \\
\hline Inadequate analgesia & 6 \\
Inadequate analgesia + hypotension & 2 \\
Inadequate analgesia + paresthesia & $\mathrm{I}$ \\
Inadequate analgesia + motor block & $\mathrm{I}$ \\
Hypotension & 7 \\
Hypotension + paresthesia & 4 \\
Hypotension + motor block & 2 \\
Motor block & 7 \\
Motor block + paresthesia & 1 \\
Paresthesia & 3 \\
Site infection & 3 \\
Site infection + oozy & 2 \\
Epidural hematoma & I \\
\hline
\end{tabular}

one of eight categories of morbid endpoints in individual systems (respiratory failure) occurred less frequently in patients managed with epidural techniques (23\% vs $30 \%$; $P=0.02)$. The authors concluded that most adverse morbid outcomes in high-risk patients undergoing major abdominal surgery were not reduced by the use of combined epidural and general anesthesia and postoperative epidural analgesia. However, the improvement in analgesia, reduction in respiratory failure, and low risk of serious adverse consequences suggest that many high-risk patients undergoing major intra-abdominal surgery will gain substantial benefit from combined intraoperative general and epidural anesthesia, with epidural analgesia continuing postoperatively. Afterwards, the same data were re-analyzed and subgroups were created. ${ }^{12}$ The conclusions suggest similar concepts: no differences were found in outcome between epidural and control groups in subgroups at increased risk of respiratory or cardiac complications or undergoing aortic surgery, or in a subgroup with failed epidural block (all $P>0.05$ ). A small reduction was found in the duration of postoperative ventilation (geometric mean [standard deviation; SD]: control group 0.3 [6.5] hours vs epidural group 0.2 [4.8] hours; $P=0.048$ ). No differences were found in length of 
stay in intensive care or in the hospital. There was no relationship between frequency of use of epidural analgesia in routine practice outside the trial and benefit from epidural analgesia in the trial.

Epidural analgesia after abdominal surgery has been reported to be more expensive; while epidural analgesia provided superior analgesia, it incurred approximately $80 \%$ higher costs than patient-controlled analgesia. ${ }^{13}$

Fast-track recovery programs encourage the use of epidurals; however, associated practical issues include theatre delays due to time required for insertion of epidurals and postoperative problems with analgesia at the time of cessation or failure of epidurals, and hypotensive episodes. ${ }^{14}$ Brodner et $\mathrm{al}^{4}$ reported that, although personal supervision was higher for patients receiving epidural analgesia, cost analysis revealed that final savings would obviate the need for 433 days of intensive care unit stay over 1 year. The authors concluded that epidural analgesia has a place in optimal perioperative pain management; however, it is more time consuming than intravenous patient-controlled analgesia.

Several studies in abdominal surgery have shown pain management with an epidural to shorten length of hospital stay. Morimoto et $\mathrm{al}^{15}$ found a shorter length of hospitalization after proctocolectomy with ileal pouch-anal canal anastomosis in patients receiving epidural fentanyl than in controls receiving systemic morphine. Another group of authors ${ }^{16}$ described a shortening of length of stay for patients undergoing laparoscopic colectomies with epidural (2.8 days) versus systemic morphine (3.9 days).

Our results suggest that epidural analgesia generates an increase in length of hospital stay that is not related to confounding factors; a significant percentage of patients undergoing epidural analgesia required a patient-controlled analgesia rescue, and theatre delays, as well as technical problems related to epidural insertion, were relevant issues. The major putative mechanisms by which regional block might reduce the risk of cancer recurrence include decreasing the surgical stress response, reducing the requirement for volatile anesthesia, and obviating the need for opioid administration. Previous data on major abdominal surgery for cancer have been analyzed in the long-term results of randomized controlled clinical trials in Australia, New Zealand, and Asia, results of which were recently published. ${ }^{17} \mathrm{~A}$ total of 503 patients undergoing potentially curative surgery for cancer were enrolled in the study. The results show a median time to recurrence of cancer or death was 2.8 (95\% confidence interval [CI] 0.7-8.7) years in the control group and $2.6(0.7-8.7)$ years in the epidural group $(P=0.61)$. Recurrence-free survival was similar in both epidural and control groups (hazard ratio $0.95,95 \%$ CI $0.76-1.17$; $P=0.61)$. Myles et $\mathrm{al}^{17}$ concluded that the use of epidural block in abdominal surgery for cancer is not associated with improved cancer-free survival. The failure rate in our study is attributable to the service being provided by anesthetists training in a teaching hospital. The practice of using epidurals with RRP has largely been abandoned in our center for the aforementioned reasons.

We state a higher risk of recurrence after RRP under epidural analgesia as shown by our results. The survival data results are controversial for two main reasons. First, our series was performed in a tertiary teaching hospital, wherein most of the epidurals and RRPs were performed by training anesthetists and urologists and so, for example, the rate of positive margins in the patients with a PSA recurrence after RRP is actually higher than expected for a non-teaching center. Second, the number of patients in our series with a recurrence after epidural is quite low.

Our results appear to contradict the results obtained by Biki et a ${ }^{18}$ regarding biochemical recurrence. These authors stated that due to immunologic responses related to general versus epidural anesthesia, they observed a reduction of PSA recurrences in men receiving epidural analgesia. Theoretically, since epidural anesthesia is believed to confer a reduced stress response, ${ }^{8}$ the human body is able to fight against residual cancer cells, resulting in a reduced PSA recurrence rate. However, the cohort of patients in the study by Biki et al was unusual in that they reported a $61 \%$ rate of positive margins $(62 / 102)$ in the epidural group - greater than the non-epidural group at $48 \%(69 / 123)$. This raises the possibility of adjuvant treatment reducing PSA recurrence, which was not reported. Further, since no clear description of patients' demographic characteristics such as TNM classification of malignant tumors were provided at a sub-group level, then some confounding factors (eg, adjuvant treatment) should be taken into account when considering their overall data on PSA recurrence. More recently, Tsui et a ${ }^{19}$ concluded, in a shorter series, that no difference was observed between the epidural and control groups in disease-free survival at a median follow-up of 4.5 years. Furthermore, Wuethrich et $\mathrm{al}^{20}$ finalize with the same conclusion: no significant difference was found between general anesthesia plus postoperative ketorolac-morphine analgesia and general anesthesia plus intraoperative and postoperative thoracic epidural analgesia in biochemical recurrence-free survival, cancer-specific survival, or overall survival. 


\section{Conclusion}

Previous studies have established that postoperative epidural analgesia has a superior analgesic effect to patient-controlled analgesia. However, results from our database have suggested an increased length of hospital stay, as well as technical problems related to the epidural, or an increase in PSA recurrence, indicating that epidurals are probably not the best analgesia for patients undergoing RRP.

There is a need for large randomized controlled trials to determine the ability of epidural analgesia to alter disease recurrence rates following RRP. However, with minimally invasive techniques becoming more widespread and epidural analgesia being used less often, this study may never be undertaken.

\section{Disclosure}

The authors report no conflicts of interest in this work.

\section{References}

1. Baade PD, Coory MD, Aitken JF. International trends in prostate-cancer mortality: the decrease is continuing and spreading. Cancer Causes Control. 2004;15:237-241.

2. Damber JE, Aus G. Prostate cancer. Lancet. 2008;371(9625): 1710-1721.

3. van Poppel H, Joniau S. Open radical prostatectomy. In: Bolla M, van Poppel H, editors. Management of Prostate Cancer: A Multidisciplinary Approach. Berlin: Springer-Verlag; 2012:93-103.

4. Brodner G, Mertes N, Buerkle H, Marcus MA, Van Aken H. Acute pain management: analysis, implications and consequences after prospective experience with 6349 surgical patients. Eur J Anaesthesiol. 2000; 17:566-575

5. Rigg JR, Jamrozik K, Myles PS, et al; MASTER Anaethesia Trial Study Group. Epidural anaesthesia and analgesia and outcome of major surgery: a randomised trial. Lancet. 2002;359:1276-1282.

6. Bar-Yosef S, Melamed R, Page GG, Shakhar G, Shakhar K, Ben-Eliyahu S. Attenuation of the tumor-promoting effect of surgery by spinal blockade in rats. Anesthesiology. 2001;94:1066-1073.
7. Exadaktylos AK, Buggy DJ, Moriarty DC, Mascha E, Sessler DI. Can anesthetic technique for primary breast cancer surgery affect recurrence or metastasis? Anesthesiology. 2006;105:660-664.

8. D'Amico AV, Whittington R, Malkowicz SB, et al. Biochemical outcome after radical prostatectomy, external beam radiation therapy, or interstitial radiation therapy for clinically localized prostate cancer. JAMA. 1998;280:969-974.

9. Ballantyne JC. Does epidural analgesia improve surgical outcome? $\mathrm{Br}$ J Anaesth. 2004;92:4-6.

10. Morgan M, Smith N, Thomas K, Murphy DG. Is Clavien the new standard for reporting urological complications? BJU Int. 2009;104: 434- 436.

11. Rodgers A, Walker N, Schug S, et al. Reduction of postoperative mortality and morbidity with epidural or spinal anaesthesia: results from overview of randomised trials. BMJ. 2000;321:1493.

12. Peyton PJ, Myles PS, Silbert BS, Rigg JA, Jamrozik K, Parsons R. Perioperative epidural analgesia and outcome after major abdominal surgery in high-risk patients. Anesth Analg. 2003;96:548-554.

13. Huang N, Cunningham F, Laurito CE, Chen C. Can we do better with postoperative pain management? Am J Surg. 2001;182:440-448.

14. Gendall KA, Kennedy RR, Watson AJ, Frizelle FA. The effect of epidural analgesia on postoperative outcome after colorectal surgery. Colorectal Dis. 2007;9:584-598; discussion 598-600.

15. Morimoto H, Cullen JJ, Messick JM Jr, Kelly KA. Epidural analgesia shortens postoperative ileus after ileal pouch-anal canal anastomosis Am J Surg. 1995;169:79-82; discussion 82-83.

16. Senagore AJ, Whalley D, Delaney CP, Mekhail N, Duepree HJ, Fazio VW. Epidural anesthesia-analgesia shortens length of stay after laparoscopic segmental colectomy for benign pathology. Surgery. 2001;129:672-676.

17. Myles PS, Peyton P, Silbert B, Hunt J, Rigg JR, Sessler DI; ANZCA Trials Group Investigators. Perioperative epidural analgesia for major abdominal surgery for cancer and recurrence-free survival: randomised trial. BMJ. 2011;342:d1491

18. Biki B, Mascha E, Moriarty DC, Fitzpatrick JM, Sessler DI, Buggy DJ. Anesthetic technique for radical prostatectomy surgery affects cancer recurrence: a retrospective analysis. Anesthesiology. 2008;109:180-187.

19. Tsui BC, Rashiq S, Schopflocher D, et al. Epidural anesthesia and cancer recurrence rates after radical prostatectomy. Can J Anaesth. 2010;57:107-112.

20. Wuethrich PY, Hsu Schmitz SF, Kessler TM, et al. Potential influence of the anesthetic technique used during open radical prostatectomy on prostate cancer-related outcome: a retrospective study. Anesthesiology. 2010;113:570-576.
Research and Reports in Urology

\section{Publish your work in this journal}

Research and Reports in Urology is an international, peer-reviewed, open access journal publishing original research, reports, editorials, reviews and commentaries on all aspects of adult and pediatric urology in the clinic and laboratory including the following topics: Pathology, pathophysiology of urological disease; Investigation and treatment of

\section{Dovepress}

urological disease; Pharmacology of drugs used for the treatment of urological disease. The manuscript management system is completely online and includes a very quick and fair peer-review system, which is all easy to use. Visit http://www.dovepress.com/testimonials.php to read real quotes from published authors. 